Article

\title{
Fluorescent Nanosensor Based on Molecularly Imprinted Polymers Coated on Graphene Quantum Dots for Fast Detection of Antibiotics
}

\author{
Tongchang Zhou, Arnab Halder ${ }^{(1)}$ and Yi Sun * \\ Department of Micro- and Nanotechnology, Technical University of Denmark, Ørsteds Plads, \\ DK-2800 Kgs Lyngby, Denmark; tongz@nanotech.dtu.dk (T.Z.); arhal@nanotech.dtu.dk (A.H.) \\ * Correspondence: sun.yi@nanotech.dtu.dk; Tel.: +45-4525-6319
}

Received: 13 July 2018; Accepted: 3 September 2018; Published: 5 September 2018

\begin{abstract}
In this work, we developed a novel fluorescent sensor by combining molecularly imprinted polymers (MIPs) with graphene quantum dots (GQDs) for the determination of tetracycline (TC) in aqueous samples. Firstly, we developed a one-pot green method to synthesize GQDs as the fluorescent probes. GQDs with carboxyl groups or amino groups were fabricated. It was found that carboxyl groups played an important role in the fluorescence quenching. Based on these findings, the GQDs-MIPs microspheres were prepared using a sol-gel process. GQDs-MIPs showed strong fluorescent emission at $410 \mathrm{~nm}$ when excited at $360 \mathrm{~nm}$, and the fluorescence was quenched in the presence of TC. Under optimum conditions, the fluorescence intensity of GQDs-MIPs decreased in response to the increase of TC concentration. The linear rage was from 1.0 to $10^{4} \mu \mathrm{g} \cdot \mathrm{L}^{-1}$, and the limit of detection was determined to be $1 \mu \mathrm{g} \cdot \mathrm{L}^{-1}$. The GQDs-MIPs also demonstrated high selectivity towards TC. The fluorescent sensor was successfully applied for the detection of TC in real spiked milk samples.
\end{abstract}

Keywords: graphene quantum dots; molecularly imprinted polymers; tetracycline; fluorescence quenching

\section{Introduction}

Tetracycline (TC) is a kind of antibiotic commonly used in food-producing animals due to its broad-spectrum activity as well as its low cost. It also works as a growth promoter in animals. Recently, TC residues in food such as milk, eggs and meats have attracted a lot of attention, since its residues in food products can accumulate in human bodies through the food chain, and cause serious side effects such as dizziness, muscle pain or headache [1]. The overuse of these antibiotics is also believed to be responsible for the formation of antibiotic-resistant bacteria strains [2,3]. As such, the European Union has set the maximum residue limit (MRL) of TC in milk to be $100 \mu \mathrm{g} \cdot \mathrm{L}^{-1}$, while the U.S. Food and Drug Administration (FDA)'s regulation is $300 \mathrm{ppb}$ (around $300 \mu \mathrm{g} \cdot \mathrm{L}^{-1}$ ) [4]. Conventionally, analytical techniques such as UV, HPLC or other techniques have been used to detect TC [5-7]. However, high cost and complicated operation procedures have limited their applications. Thus, a fast and facile method is needed to monitor the residue of TC in food samples.

Molecular imprinting is a technique for the formation of molecularly imprinted polymers (MIPs) with tailor-made binding sites complementary to the template molecules in shape, size and functional groups [8-13]. In particular, fluorescent MIP-based sensors offer a convenient solution for analyte detection due to the high sensitivity and selectivity. They have many advantages, such as short analysis time, ease of use, and small sample volume. One simple way to prepare fluorescent MIPs is adding fluorescent monomers to MIP during synthesis $[14,15]$. However, fluorescence monomer normally involves complicated synthesis work. Quantum dots (QDs) are semiconductor nanocrystals that can 
provide narrow and tunable emission spectra [16]. Compared with organic dyes, QDs have attracted much more attention due to photochemical stability and good water dispersibility. These properties make them appropriate as fluorescent probes. MIPs' coated QDs sensors have been used for detection of amoxicillin [17], melamine [18], hemoglobin [19] and cytochrome c [20]. However, most work has used CdTe QDs, which displayed high cytotoxicity [21,22]. Therefore, environmentally friendly QDs are a better alternative and potentially safer to use in point-of-source applications.

Graphene quantum dots (GQDs) are a new class of carbon nanomaterial, which have lower toxicity and better biocompatibility than the traditional QDs [23,24]. GQDs have been directly used to detect cytochrome c [25]. By incorporating GQDs into the MIP matrix, the nanocomposites will possess both the high sensitivity of fluorescent probes and the selective recognition properties of MIPs. GQDs have been widely prepared with graphene oxide (GO) as a precursor via various methods. However, many methods for GQDs fabrication require strong acids, and long purification time. In addition, different fabrication and doping methods may result in different functional groups on the surface of GQDs, whereas the effects of functional groups on fluorescence quenching have not yet been studied [26,27]. Furthermore, although MIPs-coated GQDs have been reported as a nanosensor for detection of various compounds, detection of TC in food samples using GQDs-MIPs has seldom been demonstrated.

In our work, an efficient green synthetic strategy was applied to prepare GQDs with different functional groups in a single step. We found that surface functional groups on GQDs played an important role in the fluorescence quenching. GQDs with carboxyl groups could be quenched by TC, while no quenching was observed for GQDs with amino groups. MIPs coated on GQDs with carboxyl groups were synthesized using eco-friendly reaction solvent (mixture of water and ethanol) and were used for the determination of template TC with high sensitivity. The relative fluorescence intensity of GQDs-MIPs decreased linearly with increasing TC in the concentration range of $1-10^{4} \mu \mathrm{g} \cdot \mathrm{L}^{-1} \mathrm{with}$ a detection limit of $1 \mu \mathrm{g} \cdot \mathrm{L}^{-1}$. The GQDs-MIPs were also successfully applied to detect spiked TC in milk samples and gave recoveries from $85.3 \%$ to $103.3 \%$ with relative standard deviations of $3.7 \%-7.2 \%$. These results demonstrated that GQDs-MIPs could potentially be used as a simple and fast responding fluorescence probe for sensitive and selective determination of TC.

\section{Materials and Methods}

\subsection{Materials}

All reagents were of analytical or HPLC grade and were used as received. Aminopropyltriethoxysilane (APTES), Tetraethoxysilane (TEOS), Ammonium hydroxide solution (28\%-30\%), hydrogen peroxide $\left(\mathrm{H}_{2} \mathrm{O}_{2}, 30 \%\right)$, Gentamicin (GM) and amoxicillin (AM) and tetracycline (TC) were obtained from Sigma Aldrich. Nunclon 96-well flat-bottom black microwell plates were purchased from Thermo scientific, DK. 0.1\% milk (Arla) was bought from a local supplier. The water used in the experiment was obtained from a Millipore (MilliQ) purification system.

\subsection{Synthesis of Two Kinds of Graphene Quantum Dots (GQDs)}

\subsubsection{Synthesis of Carboxylic Acid Functionalized Graphene Quantum Dots (GQDs-COOH)}

To synthesize GQDs with the carbonyl group, $40 \mathrm{mg}$ of graphene oxide precursor was mixed with $30 \mathrm{~mL}$ of $\mathrm{H}_{2} \mathrm{O}_{2}(5 \%)$. Then the mixture was transferred into a $50 \mathrm{~mL}$ Teflon-made autoclave chamber. The autoclave was put in an oven at $180{ }^{\circ} \mathrm{C}$ for $2 \mathrm{~h}$. After cooling down to room temperature, the resulting solution was filtered using $0.2 \mu \mathrm{m}$ filter paper to remove unreacted graphene oxide, and the resulting filtrate was collected and stored at room temperature. 


\subsubsection{Synthesis of Amino Functionalized Graphene Quantum Dots (GQDs- $\mathrm{NH}_{2}$ )}

Forty milligrams of graphene oxide precursor was mixed with $5 \mathrm{~mL}$ of $\mathrm{H}_{2} \mathrm{O}_{2}(30 \%), 5 \mathrm{~mL}$ of $\mathrm{NH}_{4} \mathrm{OH}(28 \%-30 \%)$ and $20 \mathrm{~mL}$ of water. The mixture was transferred into a $50 \mathrm{~mL}$ Teflon-made autoclave chamber. The reaction mixture in the autoclave was kept in an oven at $180{ }^{\circ} \mathrm{C}$ for $2 \mathrm{~h}$. After cooling down to room temperature, the resulting solution was filtered using a $0.2 \mu \mathrm{m}$ filter paper, and the resulting filtrate was collected.

\subsection{Preparation of GQDs-MIPs from Sol-Gel Process}

Briefly, $15 \mathrm{~mL}$ of GQDs ( $3 \mathrm{mg}$ ) solution and $10 \mathrm{~mL}$ of ethanol were added into a flask. Then $80 \mu \mathrm{L}$ of APTES were added and stirred for $2 \mathrm{~h}$ under vigorous stirring to allow the APTES to self-assemble onto the GQDs. Template TC $(10 \mathrm{mg})$ was then dissolved in ultrapure water $(10 \mathrm{~mL})$ and added to the above solution. After stirring for $15 \mathrm{~min}, 100 \mu \mathrm{L}$ of ammonia hydroxide solution (25\%) was added, then $100 \mu \mathrm{L}$ of TEOS and $10 \mathrm{~mL}$ of ethanol were added drop by drop. The reaction mixture was stirred at room temperature for $24 \mathrm{~h}$.

The final products GQDs-MIPs were collected by centrifugation, then washed thoroughly with ethanol and MilliQ water. After each washing step, the supernatant was measured by UV to check whether there was TC residue in the solution. Typically the GQDs-MIPs were washed five times in order to completely remove the TC template. Non-imprinted particles (GQDs-NIPs) as a control were prepared similarly, except that the template was not added.

\subsection{Characterization of GQDs-MIPs}

TEM images were taken using a Tecnai T20 G2 (FEI, Hillsboro, OR, USA) transmission electron microscope. IR spectra were taken using a Spectrum 100 (PerkinElmer, Waltham, MA, USA). X-ray photoelectron spectroscopy (XPS) analysis was carried out by Thermo Scientific ${ }^{\mathrm{TM}} \mathrm{K}$-Alpha+ ${ }^{\mathrm{TM}}$ XPS System with an Al K-Alpha (1486 eV) X-ray source.

\subsection{Fluorescence Measurement of GQDs-MIPs}

TC with different concentrations $\left(2,20,200,2000\right.$ and $\left.2 \times 10^{4} \mu \mathrm{g} \cdot \mathrm{L}^{-1}, 150 \mu \mathrm{L}\right)$ and GQDs-MIPs solution $\left(150 \mu \mathrm{L}, 1 \mathrm{mg} \cdot \mathrm{mL}^{-1}\right)$ were sequentially injected into each well of a Nunclon 96-well flat-bottom black microplate. Then, the fluorescence spectra were measured with an excitation wavelength of $360 \mathrm{~nm}$, using a Spark ${ }^{\circledR}$ multimode microplate reader (Tecan, Sweden). The fluorescent intensity at $410 \mathrm{~nm}$ was used for analysis.

For detection of TC in real samples, TC with the concentrations of 2, 20, 200, 2000 and $2 \times 10^{4} \mu \mathrm{g} \cdot \mathrm{L}^{-1}$ were spiked into the diluted milk (Arla $0.1 \%$, diluted 200 folds). For each concentration, three samples were tested to get the standard deviation. The measurement was taken in the same manner as described above.

\section{Results and Discussion}

\subsection{Green Synthesis of GQDs}

It is of great importance to explore a mild, clean, and highly efficient approach for the synthesis of GQDs. Generally, GQDs were synthesized by traditional methods such as electrochemical exfoliation [28], chemical oxidation [29] and microwave-assisted methods [30]. Strong acids and chemicals are often involved as oxidants in most approaches. Some amount of chemicals are left as residues in the resulted GQDs solution, which need repeated washing steps to get pure GQDs. Herein, we introduced a new green and rapid preparation approach for GQDs using a low amount of hydrogen peroxide as an oxidant, as shown in Figure 1. As a degrading agent for graphene, $\mathrm{H}_{2} \mathrm{O}_{2}$ could cut down graphene into smaller sized graphene-based QDs. The byproducts are only $\mathrm{H}_{2} \mathrm{O}$ and $\mathrm{CO}_{2}$, and a post purification step is not needed. 


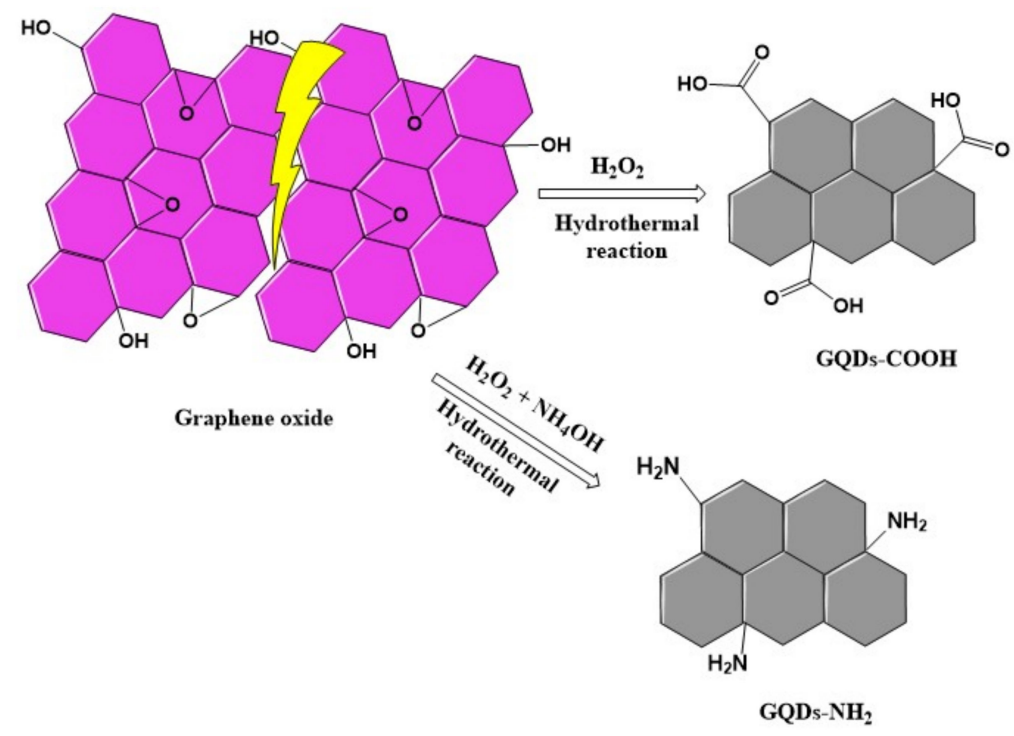

Figure 1. Illustration of the preparation of GQDs from graphene oxide.

\subsection{Comparison of GQDs with Different Functional Groups}

As carbon-based materials, GQDs have excellent properties, such as high surface area and low toxicity. In addition, surface functional groups can effectively tune their properties, which can extend their application areas. In this work, we successfully prepared GQDs with two kinds of functional groups. Figure 2a shows FT-IR spectra of the functional groups on the GQDs. The sharp peak appearing at $1720 \mathrm{~cm}^{-1}$ and $1226 \mathrm{~cm}^{-1}$ belonged to the $\mathrm{C}=\mathrm{O}$ stretching and $\mathrm{C}-\mathrm{O}$ stretching of carboxylic acid, respectively. In the meantime, a band appeared at $1576 \mathrm{~cm}^{-1}$ and was assigned to C-N stretching while the band appearing at $1291 \mathrm{~cm}^{-1}$ was assigned to the mixed vibration of C-N stretching and N-H bending. The detailed surface chemical bonding nature of GQDs-COOH and GQDs- $\mathrm{NH}_{2}$ were evaluated using XPS. As shown in Figure $2 b$, both of GQDs-COOH and GQDs- $\mathrm{NH}_{2}$ showed $\mathrm{C} 1 \mathrm{~s}$ and $\mathrm{O} 1 \mathrm{~s}$ peaks at around $286 \mathrm{eV}$ and $532 \mathrm{eV}$. However, only a new peak was observed for GQDs- $\mathrm{NH}_{2}$ at around $400 \mathrm{eV}$, which was due to the presence of nitrogen. The $\mathrm{C} 1 \mathrm{~s}$ spectra of GQDs-COOH was further deconvoluted into two peaks with binding energies at 284.9 and $288.7 \mathrm{eV}$, which correspond to sp2 and sp3 hybridized carbon $(\mathrm{C}-\mathrm{C} / \mathrm{C}=\mathrm{C})$ and $\mathrm{O}-\mathrm{C}=\mathrm{O}$ components respectively (Figure 2c).
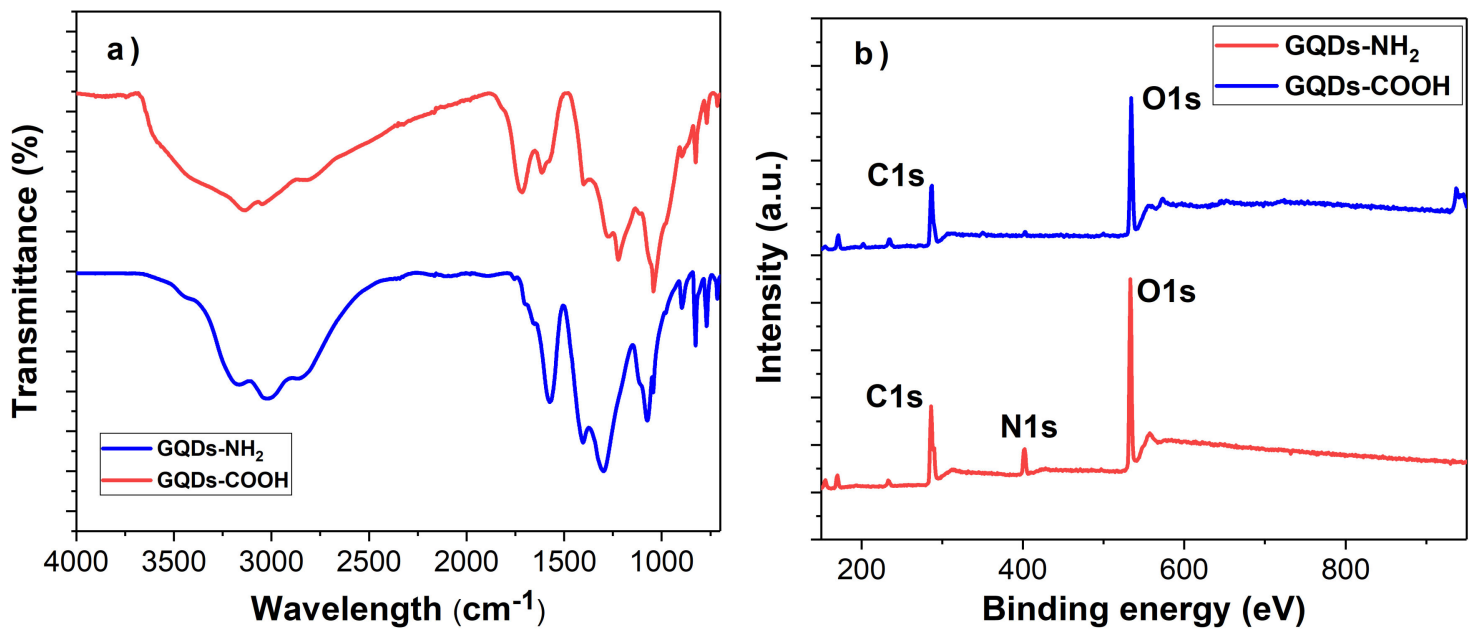

Figure 2. Cont. 


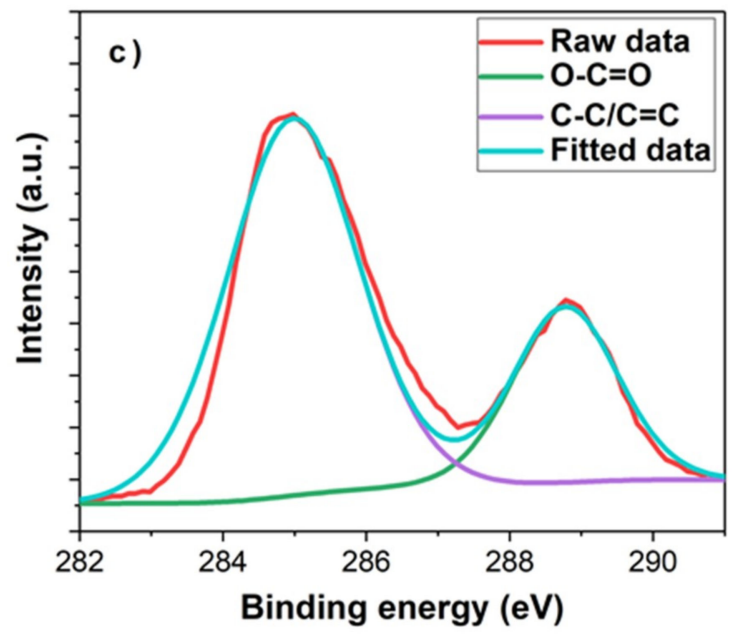

Figure 2. (a) FTIR of two types of GQDs. (b) XPS survey spectra for GQDs-COOH and GQDs-NH2. (c) The high resolution deconvoluted $\mathrm{C}$ 1s spectra for GQDs-COOH.

To compare these GQDs with different functional groups, we tested the fluorescence emission spectra of GQDs with the addition of TC solutions. Quenching of GQDs-COOH was observed at the concentration at and above $10^{4} \mu \mathrm{g} \cdot \mathrm{L}^{-1}$, as shown in Figure 3a. In comparison, the fluorescence of GQDs- $\mathrm{NH}_{2}$ could not be quenched in the presence of TC. Quenching requires molecular contact and energy transfer between the fluorophore and quencher. Here GQDs-COOH was negatively charged while GQDs- $\mathrm{NH}_{2}$ was positively charged. Since ammonium groups of TC were positively charged, electrostatic interaction occurred between GQDs-COOH and the template TC, which resulted in the apparent nonradiative annihilation, as reported by Chao [31]. This was further proved by the zeta potential of GQDs-COOH, which was found to be $-27.6 \mathrm{mV}$ in water. Therefore, MIPs based on GQDs-COOH were applied for further experiments.

To study the quenching mechanism, the absorption spectra of GQDs-COOH in the presence and absence of TC were measured as illustrated in Figure 3b. It was observed that the absorbance of GQDs changed with the addition of TC, indicating the interaction between GQDs and TC induced static quenching, as static quenching affects the absorption spectrum of the quenching molecule.

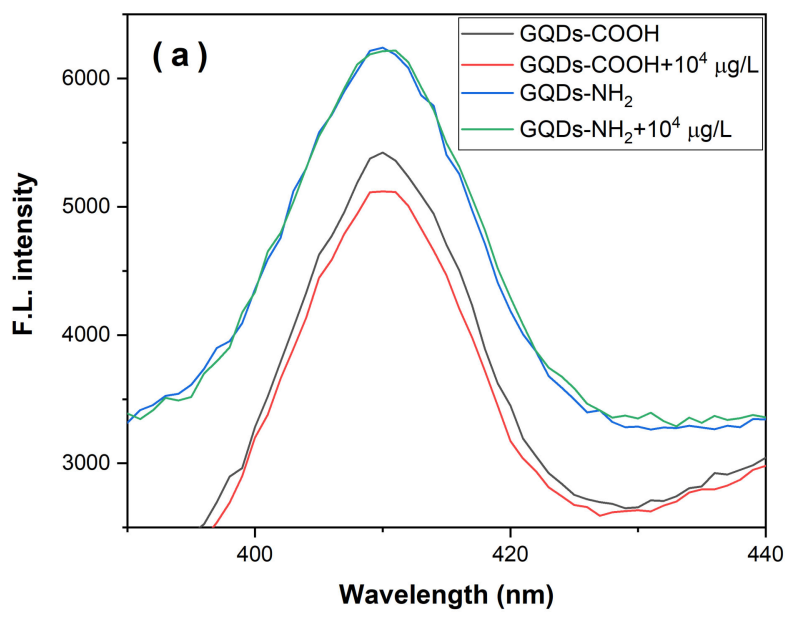

Figure 3. Cont. 


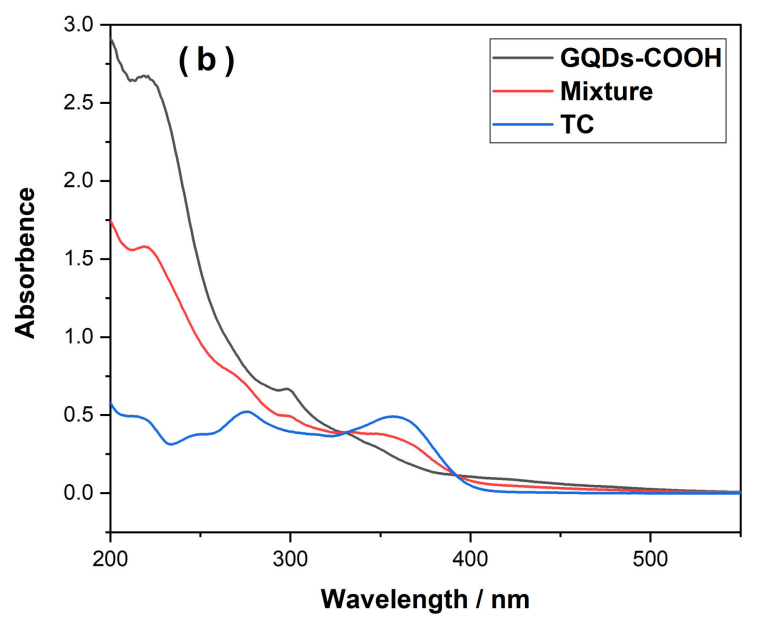

Figure 3. (a) Fluorescence emission spectra of GQDs with different functional groups with addition of TC in water. (b) UV spectrum of GQDs-COOH, TC and mixture.

\subsection{TEM of GQDs-MIP and GQDs}

MIP and reference NIP were successfully synthesized by sol-gel process. The morphology and size distribution of GQDs, GQDs-MIPs and GQDs-NIPs were investigated by TEM, as illustrated in Figure 4. The GQDs had a good size distribution around $5 \mathrm{~nm}$ while both the GQDs-MIPs and GQDs-NIPs exhibited a size distribution with diameters in the range of 200-300 nm. After the coating of MIPs on GQDs, it is clear that the particle's diameters increased significantly.
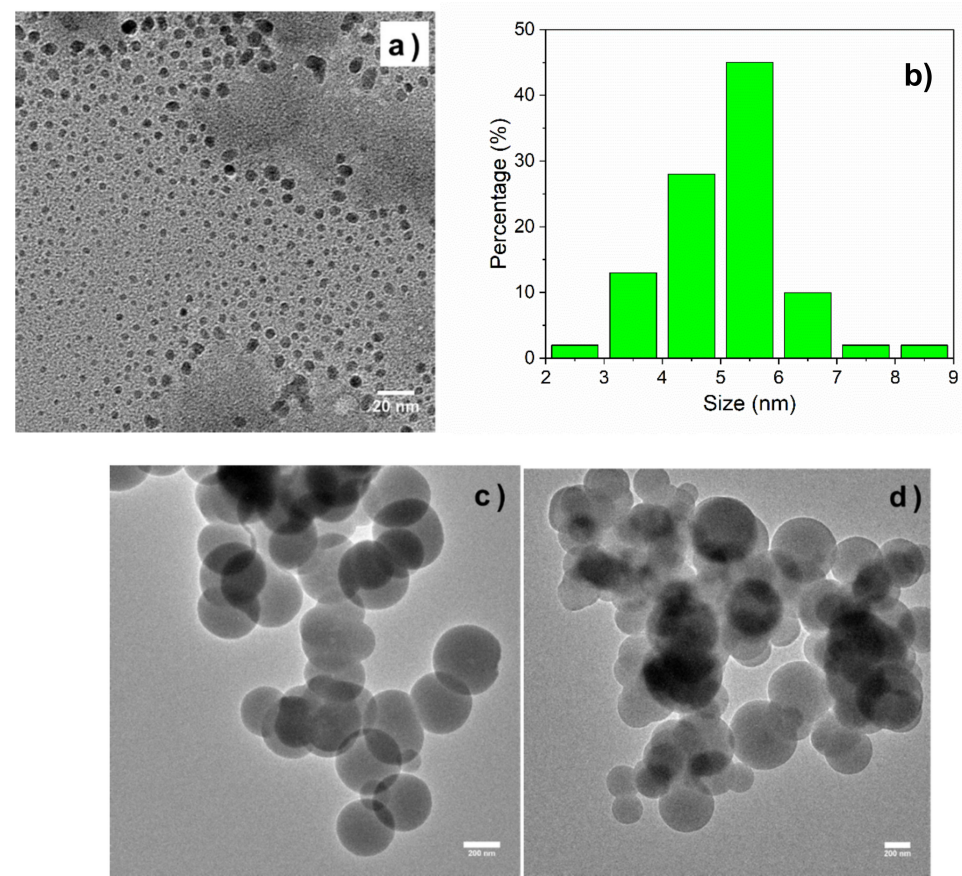

Figure 4. TEM images of GQDs (a), GQDs-MIPs (c), and GQDs-NIPs (d). The hydrodynamic size distribution of GQDs (b).

\subsection{Determination of TC Using GQDs-MIPs}

To study the recognition ability of GQDs-MIPs, the fluorescence intensity of GQDs-MIPs and GQDs-NIPs were investigated at different concentrations of TC, which are shown in Figure 5a,b. Although both GQDs-MIPs and NIPs showed fluorescence quenching, the intensity of GQDs-MIPs 
decreased more significantly in response to the increase of TC concentration. It is clear that specific cavities of MIPs resulted in the higher quenching efficiency of GQDs-MIPs. The results showed that the GQDs-MIPs exhibited a linear quenching for the TC detection in the range of $1.0-10^{4} \mu \mathrm{g} \cdot \mathrm{L}^{-1}$, and the limit of detection for TC was determined to be $1 \mu \mathrm{g} \cdot \mathrm{L}^{-1}$ under optimal conditions (Figure 5c). Normally, a certain incubation time is needed for sufficient interactions between analytes and GQDs-MIPs. It is worth noting that here all tests were measured right after TC was mixed with GQDs-MIPs without incubation, suggesting that the response time of the developed sensor was very short and the quenching occurred rapidly. This is very helpful for fast detection of TC in real samples.
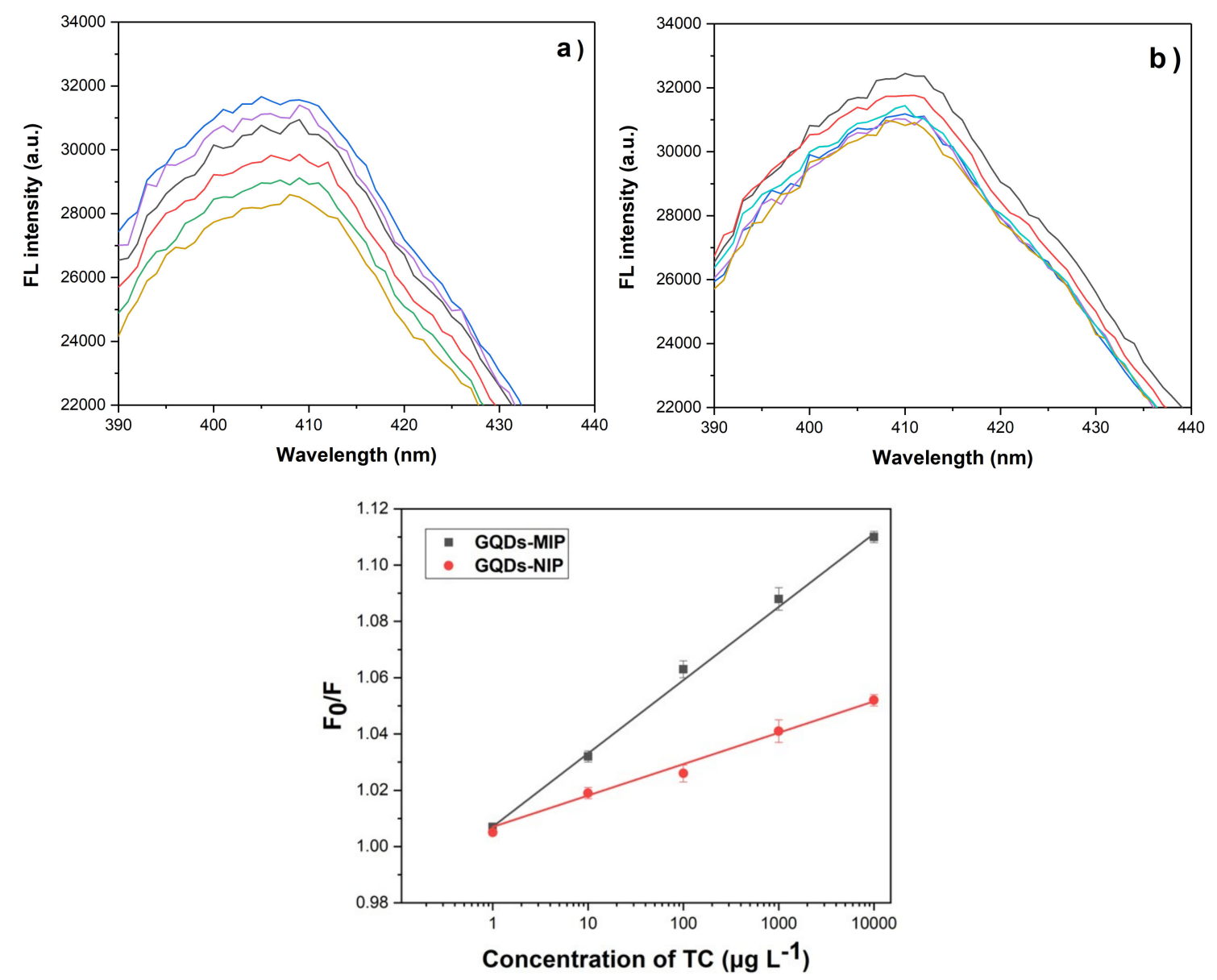

Figure 5. Fluorescence emission spectra of GQDs-MIPs (a) and GQDs-NIPs (b) with the addition of various amounts of TC in water. $\mathrm{F}_{0}$ and $\mathrm{F}$ represent the fluorescent intensities before and after the addition of TC solution, respectively.

\subsection{Selectivity of GQDs-MIPs}

The selectivity of the GQDs-MIPs was further investigated by fluorescence quenching response to analog analytes. One interference compound was doxycycline (DOX), a synthetic antibiotic derived from TC. The chemical differences between them are in the substitutes of some carbon atoms, as shown in Figure 6. Because of the effectiveness of molecularly imprinting, we found that the template TC bound more strongly to the imprinted sites than DOX, and more significant changes in the fluorescence intensity of GQDs-MIPs were observed. Due to a big structure difference, the quenching of GQDs-MIPs by Gentamicin (GM) and amoxicillin (AM) were quite small. The above results revealed that GQDs-MIPs exhibited a selective recognition and excellent fluorescence quenching response toward TC. As the molecular size and shape are very similar between TC and DOX, the position of the functional groups is the major dominating factor for a specific fluorescence quenching response. 

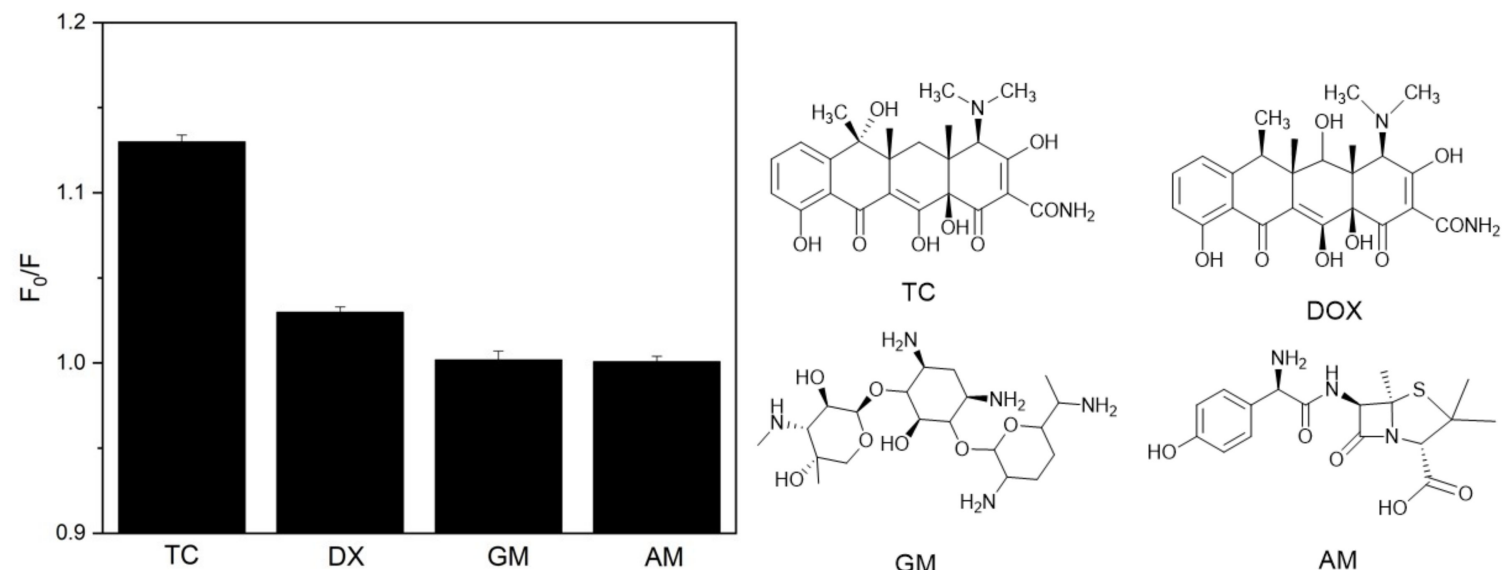

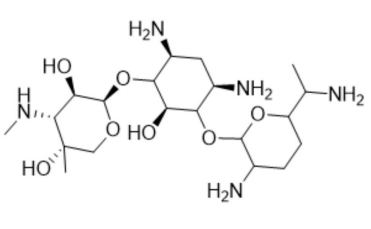

GM

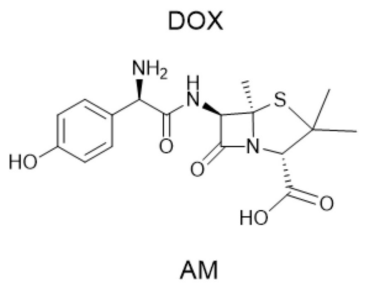

Figure 6. Selective adsorption of TC, DOX, GM and AM by GQDs-MIPs in water. The concentration of all compounds was $10^{4} \mu \mathrm{g} \mathrm{L}-1$.

\subsection{Real Sample Analysis}

To further demonstrate the selectivity and sensitivity of GQDs-MIPs, detection and quantification of TC in real milk samples were conducted. According to the results shown in Table 1, the spiked recoveries of TC were ranging from $85.3 \%$ to $103.3 \%$, which suggested that the combination of GQDs and MIPs was an effective method for analyzing antibiotic residues in complex food samples.

Table 1. Recovery of TC from spiked milk samples.

\begin{tabular}{ccc}
\hline Spiked Amount $\left(\boldsymbol{\mu g} \cdot \mathbf{L}^{-\mathbf{1}}\right)$ & Measured Amount $\left(\boldsymbol{\mu g} \cdot \mathbf{L}^{-\mathbf{1}}\right)$ & Recovery $(\%)$ \\
\hline 1 & $0.98 \pm 0.049$ & $98 \pm 4.9$ \\
10 & $9.22 \pm 0.57$ & $92.2 \pm 5.7$ \\
100 & $103.3 \pm 3.7$ & $103.3 \pm 3.7$ \\
$10^{3}$ & $(9.74 \pm 0.72) \times 10^{2}$ & $97.4 \pm 7.2$ \\
$10^{4}$ & $(8.53 \pm 0.61) \times 10^{3}$ & $85.3 \pm 6.1$ \\
\hline
\end{tabular}

\section{Conclusions}

Nowadays, rapid and efficient detection methods for the trace amounts of antibiotics in food samples are in urgent need for food security screening. In this work, GQDs were fabricated via a simple and green synthesis approach, and the effect of functional groups on fluorescence quenching was investigated. A GQDs-MIPs-based fluorescence nanosensor was then successful prepared by the simple sol-gel method. The results showed fast detection and selectivity. Compared with natural bioassay, the fluorescent sensor has higher stability and lower cost. The work suggested that GQDs-MIPs could be widely used to screen and analyze TC in complex food samples.

Author Contributions: Conceptualization, Y.S., T.Z. and A.H.; Methodology, T.Z and A.H.; Formal Analysis, T.Z. and A.H.; Writing_-Original Draft Preparation, T.Z.; Writing-Review \& Editing, T.Z., A.H. and Y.S.; Supervision, Y.S.; Project Administration, Y.S.; Funding Acquisition, Y.S.

Acknowledgments: This work was financially supported by the Villum Fonden, Denmark, Project No. 13153.

Conflicts of Interest: The authors declare no conflict of interest.

\section{References}

1. Chopra, I.; Roberts, M. Tetracycline Antibiotics: Mode of Action, Applications, Molecular Biology, and Epidemiology of Bacterial Resistance. Microbiol. Mol. Biol. Rev. 2001, 65, 232-260. [CrossRef] [PubMed] 
2. Akiba, T.; Koyama, K.; Ishiki, Y. On the mechanism of the development of multiple-drug-resistant clones of Shigella. Jpn. J. Microbiol. 1960, 4, 219-227. [CrossRef] [PubMed]

3. Roberts, M.C. Tetracycline Therapy: Update. Clin. Infect. Dis. 2003, 36, 462-467. [CrossRef] [PubMed]

4. Podhorniak, L.V.; Leake, S.; Schenck, F.J. Stability of tetracycline antibiotics in raw milk under laboratory storage conditions. J. Food Prot. 1999, 62, 547-548. [CrossRef] [PubMed]

5. Chen, L.; Liu, J.; Zeng, Q.; Wang, H.; Yu, A.; Zhang, H.; Ding, L. Preparation of magnetic molecularly imprinted polymer for the separation of tetracycline antibiotics from egg and tissue samples. J. Chromatogr. A 2009, 1216, 3710-3719. [CrossRef] [PubMed]

6. Jing, T.; Gao, X.D.; Wang, P.; Wang, Y.; Lin, Y.F.; Hu, X.Z.; Hao, Q.L.; Zhou, Y.K.; Mei, S.R. Determination of trace tetracycline antibiotics in foodstuffs by liquid chromatography-tandem mass spectrometry coupled with selective molecular-imprinted solid-phase extraction. Anal. Bioanal. Chem. 2009, 393, 2009-2018. [CrossRef] [PubMed]

7. Lv, Y.K.; Zhao, C.X.; Li, P.; He, Y.D.; Yang, Z.R.; Sun, H.W. Preparation of doxycycline-imprinted magnetic microspheres by inverse-emulsion suspension polymerization for magnetic dispersion extraction of tetracyclines from milk samples. J. Sep. Sci. 2013, 36, 2656-2663. [CrossRef] [PubMed]

8. Shen, X.; Svensson Bonde, J.; Kamra, T.; Bülow, L.; Leo, J.C.; Linke, D.; Ye, L. Bacterial imprinting at pickering emulsion interfaces. Angew. Chem Int. Ed. 2014, 53, 10687-10690. [CrossRef] [PubMed]

9. Bedwell, T.S.; Whitcombe, M.J. Analytical applications of MIPs in diagnostic assays: Future perspectives. Anal. Bioanal. Chem. 2016, 408, 1735-1751. [CrossRef] [PubMed]

10. Liu, L.; Yang, K.; Zhang, L.; Zhang, Y. Protein-imprinted material for the treatment of antibiotic-resistant bacteria. Sci. Bull. 2016, 61, 1890-1891. [CrossRef]

11. Chen, L.; Wang, X.; Lu, W.; Wu, X.; Li, J. Molecular imprinting: Perspectives and applications. Chem. Soc. Rev. 2016, 45, 2137-2211. [CrossRef] [PubMed]

12. Rechichi, A.; Cristallini, C.; Vitale, U.; Ciardelli, G.; Barbani, N.; Vozzi, G.; Giusti, P. New biomedical devices with selective peptide recognition properties. Part 1: Characterization and cytotoxicity of molecularly imprinted polymers. J. Cell. Mol. Med. 2007, 11, 1367-1376. [CrossRef] [PubMed]

13. Morelli, I.; Chiono, V.; Vozzi, G.; Ciardelli, G.; Silvestri, D.; Giusti, P. Molecularly imprinted submicronspheres for applications in a novel model biosensor-film. Sens. Actuators B Chem. 2010, 150, 394-401. [CrossRef]

14. Wan, W.; Biyikal, M.; Wagner, R.; Sellergren, B.; Rurack, K. Fluorescent sensory microparticles that "light-up" consisting of a silica core and a molecularly imprinted polymer (MIP) shell. Angew. Chem Int. Ed. 2013, 52, 7023-7027. [CrossRef] [PubMed]

15. Ashley, J.; Feng, X.; Sun, Y. A multifunctional molecularly imprinted polymer-based biosensor for direct detection of doxycycline in food samples. Talanta 2018, 182, 49-54. [CrossRef] [PubMed]

16. Yang, Y.; Yi, C.; Luo, J.; Liu, R.; Liu, J.; Jiang, J.; Liu, X. Glucose sensors based on electrodeposition of molecularly imprinted polymeric micelles: A novel strategy for MIP sensors. Biosens. Bioelectron. 2011, 26, 2607-2612. [CrossRef] [PubMed]

17. Chullasat, K.; Nurerk, P.; Kanatharana, P.; Davis, F.; Bunkoed, O. A facile optosensing protocol based on molecularly imprinted polymer coated on CdTe quantum dots for highly sensitive and selective amoxicillin detection. Sens. Actuators B Chem. 2018, 254, 255-263. [CrossRef]

18. $\mathrm{Xu}, \mathrm{S}$.; $\mathrm{Lu}, \mathrm{H}$. One-pot synthesis of mesoporous structured ratiometric fluorescence molecularly imprinted sensor for highly sensitive detection of melamine from milk samples. Biosens. Bioelectron. 2015, 73, 160-166. [CrossRef] [PubMed]

19. Zhang, W.; He, X.W.; Chen, Y.; Li, W.Y.; Zhang, Y.K. Composite of CdTe quantum dots and molecularly imprinted polymer as a sensing material for cytochrome c. Biosens. Bioelectron. 2011, 26, 2553-2558. [CrossRef] [PubMed]

20. Li, D.-Y.; He, X.-W.; Chen, Y.; Li, W.-Y.; Zhang, Y.-K. Novel Hybrid Structure Silica/CdTe/Molecularly Imprinted Polymer: Synthesis, Specific Recognition, and Quantitative Fluorescence Detection of Bovine Hemoglobin. ACS Appl. Mater. Interfaces 2013, 5, 12609-12616. [CrossRef] [PubMed]

21. Fang, T.T.; Li, X.; Wang, Q.S.; Zhang, Z.J.; Liu, P.; Zhang, C.C. Toxicity evaluation of CdTe quantum dots with different size on Escherichia coli. Toxicol. In Vitro 2012, 26, 1233-1239. [CrossRef] [PubMed]

22. Yang, Q.; Li, J.; Wang, X.; Peng, H.; Xiong, H.; Chen, L. Strategies of molecular imprinting-based fluorescence sensors for chemical and biological analysis. Biosens. Bioelectron. 2018, 112, 54-71. [CrossRef] [PubMed]

23. Gravagnuolo, A.M.; Morales-Narváez, E.; Longobardi, S.; Da Silva, E.T.; Giardina, P.; Merkoçi, A. In situ production of biofunctionalized few-layer defect-free microsheets of graphene. Adv. Funct. Mater. 2015, 25, 2771-2779. [CrossRef] 
24. Mehrzad-Samarin, M.; Faridbod, F.; Dezfuli, A.S.; Ganjali, M.R. A novel metronidazole fluorescent nanosensor based on graphene quantum dots embedded silica molecularly imprinted polymer. Biosens. Bioelectron. 2017, 92, 618-623. [CrossRef] [PubMed]

25. Cao, L.; Li, X.; Qin, L.; Kang, S.-Z.; Li, G. Graphene quantum dots supported by graphene oxide as a sensitive fluorescence nanosensor for cytochrome c detection and intracellular imaging. J. Mater. Chem. B 2017, 5, 6300-6306. [CrossRef]

26. Zhou, X.; Wang, A.; Yu, C.; Wu, S.; Shen, J. Facile Synthesis of Molecularly Imprinted Graphene Quantum Dots for the Determination of Dopamine with Affinity-Adjustable. ACS Appl. Mater. Interfaces 2015, 7, 11741-11747. [CrossRef] [PubMed]

27. Amjadi, M.; Jalili, R. Molecularly imprinted polymer-capped nitrogen-doped graphene quantum dots as a novel chemiluminescence sensor for selective and sensitive determination of doxorubicin. RSC Adv. 2016, 6, 86736-86743. [CrossRef]

28. Li, Y.; Hu, Y.; Zhao, Y.; Shi, G.; Deng, L.; Hou, Y.; Qu, L. An electrochemical avenue to green-luminescent graphene quantum dots as potential electron-acceptors for photovoltaics. Adv. Mater. 2011, 23, 776-780. [CrossRef] [PubMed]

29. Liu, R.; Wu, D.; Feng, X.; Müllen, K. Bottom-up fabrication of photoluminescent graphene quantum dots with uniform morphology. J. Am. Chem. Soc. 2011, 133, 15221-15223. [CrossRef] [PubMed]

30. Hou, J.; Li, H.; Wang, L.; Zhang, P.; Zhou, T.; Ding, H.; Ding, L. Rapid microwave-assisted synthesis of molecularly imprinted polymers on carbon quantum dots for fluorescent sensing of tetracycline in milk. Talanta 2016, 146, 34-40. [CrossRef] [PubMed]

31. Chao, M.R.; Hu, C.W.; Chen, J.L. Comparative syntheses of tetracycline-imprinted polymeric silicate and acrylate on CdTe quantum dots as fluorescent sensors. Biosens. Bioelectron. 2014, 61, 471-477. [CrossRef] [PubMed]

(C) 2018 by the authors. Licensee MDPI, Basel, Switzerland. This article is an open access article distributed under the terms and conditions of the Creative Commons Attribution (CC BY) license (http:/ / creativecommons.org/licenses/by/4.0/). 